\title{
Directly applied low intensity direct electric current enhances peripheral nerve regeneration in rats
}

\author{
Adriana Clemente Mendonça, Cláudio Henrique Barbieri*, Nilton Mazzer \\ Laboratory of Bioengineering, Bioengineering Postgraduation Program, Department of Biomechanics, Medicine and Rehabilitation of the Locomotor \\ Apparatus, Ribeirão Preto School of Medicine, São Paulo University, Ribeirão Preto SP 14049 900, Brazil
}

Received 24 January 2003; received in revised form 26 June 2003; accepted 26 June 2003

\begin{abstract}
The influence of direct electric stimulation on nerve regeneration was studied in a model of crush injury of the sciatic nerve of rats. Forty-three rats were used and distributed in four groups according to the procedure: (1) intact nerve, inactive circuit; (2) crush injury, inactive circuit; (3) intact nerve, active circuit; (4) crush injury, active circuit. The low intensity continuous current circuit (1 $\mu \mathrm{A}$ ) was implanted in the lumbar region, the anode being fixed to the muscles proximally and the cathode below the nerve distally to the lesion site. The Sciatic Functional Index (SFI) was evaluated at weekly intervals for 3 weeks, the sciatic nerve being resected on the 21st day for histologic and morphometric studies. The SFI progressively improved and the average fiber nerve density recovered to a nearly normal value in Group 2 and increased in Group 4 compared with the control groups (1 and 3), but this was accompanied by a decreased average fiber nerve diameter. Both number and diameter of inter and intra-fascicular blood vessels increased in the stimulated nerves. We conclude that low intensity direct electric stimulation enhances nerve regeneration following a controlled nerve crush injury and increases blood supply by increasing number and diameter of vasa nervorum.
\end{abstract}

(C) 2003 Elsevier B.V. All rights reserved.

Keywords: Peripheral nerve regeneration; Sciatic nerve of rats; Crush injury; Low intensity electric stimulation; Sciatic functional index; Morphometry

\section{Introduction}

It is well known now-a-days that physics agents like electricity, magnetic field and ultrasound may positively influence the outcome of the healing process of different tissues like skin, bone, muscles and tendons and peripheral nerves (Brighton et al., 1981; Behse et al., 1990). There are many evidences that regeneration of the peripheral nerves can be accelerated by electric stimulation and a number of experimental studies have shown that the first signs of regeneration begin to appear by the third postoperative week and continue to happen for up to 90 days.

Authors are unanimous to state that it is low intensity electric currents that have a beneficial effect upon the regeneration of peripheral nerves, but neither the intensity suggested nor the material used to make the

* Corresponding author. Tel./fax: +55-16-633-7559.

E-mail address: chbarbie@fmrp.usp.br (C.H. Barbieri). electrodes very much vary from one another. As refers to intensity, while some authors used a very low current of up to $1.5 \mu \mathrm{A}$ (Pomeranz et al., 1984; Kerns et al., 1987, 1991; Beveridge and Politis, 1988; Politis et al., 1988a,b; Shen and Zhu, 1995), others used $10 \mu \mathrm{A}$ (McDevitt et al., 1987; Roman et al., 1987; Pomeranz and Campbell, 1993) or higher (Kerns et al., 1986, with $10 \mu \mathrm{A} / \mathrm{cm}^{2}$ ). The electrodes used were made of silver/ silver chloride (Kerns et al., 1987, 1991; McDevitt et al., 1987; Pomeranz and Campbell, 1993; Shen and Zhu, 1995), stainless steel (Pomeranz et al., 1984; Beveridge and Politis, 1988) or platine (Roman et al., 1987).

Evaluation of peripheral nerve regeneration in animals is usually based on data obtained mostly from morphologic, morphometric and electrophysiologic studies, functional studies seldom being used for obvious reasons. However, as early as 1942 the ability to "spread the toes" had already been pointed out as a reliable criterion to evaluate recovery of the peroneal nerve in rabbits (Gutmann and Gutmann, 1942) and this was the 
base for the development of a method of functional evaluation in rats submitted to a crush injury of the sciatic nerve and footprint analysis, called Sciatic Functional Index (SFI) (De Medinaceli et al., 1982, 1984). The SFI was later modified (Bain et al., 1989) and proved to closely match the morphometric evaluation in rats (Oliveira et al., 2001). According to the modification by Bain and colleagues, the method requires the measurement of three parameters on the rat's hind paw print, which are introduced into a specific mathematics formula. The SFI actually is a negative indicator of the degree of nerve dysfunction and varies from zero to -100 , with zero corresponding to normal function and -100 to complete dysfunction.

It was the purpose of the present investigation to study the influence of direct electric current on the regeneration of the sciatic nerve of rats, using a model of controlled severe crush injury and evaluating both functional and morphologic recovery by correlating SFI and morphometric data.

\section{Methods}

The experiment was carried out in 43 male Wistar rats with $300-350 \mathrm{~g}$ average body weight. Before and after the operation the animals were kept in individual cages and fed with specific rat chow and water ad libitum. All were given a single weight related subcutaneous dose of antibiotic (Penicillin-procaine, 400000 IU) the day before the operation. The animals were distributed into four groups according to the operative procedure:

Group 1: intact nerve, inactive circuit implanted $(n=$ 9);

Group 2: crush injury, inactive circuit implanted $(n=$ 10);

Group 3: intact nerve, active circuit implanted $(n=$ 10);

Group 4: crush injury, active circuit implanted $(n=$ 14).

The electric stimulator was built according to a preexisting model (Kerns et al., 1987; Shen and Zhu, 1995). It consisted of a circuit formed by a $1.5 \mathrm{~V}$ battery and a $1.3 \mathrm{M} \Omega$ resistor, both of small dimensions and weight, and was designed so as to deliver a constant $1 \mu \mathrm{A}$ continuous electric current. A very high resistance resistor was purposefully used to prevent virtually any influence of the resistance of the surrounding tissues on the current delivered, which was then constant. The electrodes were made with stainless steel flexible wire ( $0.65 \mathrm{~mm}$ in diameter) of medical use isolated with a blue polyethilene tube $\left(\right.$ Ethicon $\left.^{\circledR}\right)$. After welding both the resistor and electrodes in line with the battery, the circuit was embedded in epoxy resin and left to dry, following which it was embedded in silicone resin of medical use (Dow Corning ${ }^{\circledR}$ ). The circuit's final dimension was $17 \mathrm{~mm}$ in diameter, with the electrodes measuring $2 \mathrm{~cm}$ in length, with an altogether weight of $3 \mathrm{~g}$.

Before effective use in the animals, the stimulator was exhaustively tested for duration and stability of electric current deliverance and impermeability and proved to be able to deliver the $1 \mu \mathrm{A}$ direct current for over a month without suffering with immersion in water for the same period. The stimulator also resisted sterilization with ethylene oxide, which was then routinely used.

\subsection{Operative procedure}

Immediately before the operation, the rats were taught to walk on the walking track by repetitive trials and normal preoperative hind paw prints were then obtained, identified and stored. The animals were then anaesthetized with a single intraperitoneal injection of sodium pentobarbiturate (Nembutal Abbott ${ }^{\circledR}, 60 \mathrm{mg} / \mathrm{kg}$ body weight) and placed prone on the table, both front and hind paws fixed with rubber bands. The lateral aspect of the right thigh, hip and flank were then prepared as routinely, including trimming off the hair and antisepsis ( $20 \%$ iodine alcohol solution), and the operative field was protected with sterile towels. The sciatic nerve was exposed through a posterolateral longitudinal straight incision going down from the greater trochanter to the lateral condyle of the femur, followed by blunt dissection between the gluteus maximus and quadriceps muscles. The entire length of the nerve was made visible but it was only detached from the surrounding soft tissues at the level of its middle third, for production of the crush injury, which was made in a universal testing machine. A stable $15000 \mathrm{~g}$ load was then applied for $10 \mathrm{~min}$ to a $5 \mathrm{~mm}$-long intermediate segment, according to a previously devised model (Oliveira et al., 2001). The damage inflicted on the nerve by such a load was purposefully intense to make spontaneous regeneration more difficult and to facilitate comparisons between the stimulated and nonstimulated nerves. On the other hand, the lesion produced was very uniform.

Following the production of the crush injury, the sciatic nerve was carefully detached from the equipment and placed back into its original bed. The animal was taken back to the operating table and the stimulating electric circuit was implanted. The bulk comprising the silicone sealed battery and resistor was placed into the subcutaneous space developed in the flank and sutured onto the muscle below with two isolated 4/0 nylon stitches. Both electrodes were passed through a small hole made in the quadriceps muscle, which they were also sutured to with an 8 -shaped $8 / 0$ nylon stitch so as to prevent migration. This way, both electrodes were 
placed in the vicinity of the sciatic nerve: the anode (positive) was introduced and fixed with a stitch into the pyriform muscle immediately over the nerve emergence and about $8 \mathrm{~mm}$ above the crush injury site; the cathode (negative) was placed directly underneath the nerve at a point $7 \mathrm{~mm}$ below the injury site, where it was firmly fixed with one or two stitches onto the neighbor muscles so as to avoid friction against the nerve. The average distance between both electrodes was $15 \mathrm{~mm}$. As soon as the implantation of the circuit was finished, the delivered electric current amperage was checked in situ to make sure that there had been no loss and the wound was closed by layers. Some more antiseptic solution ( $20 \%$ iodine alcohol solution) was sprayed on the wound but no dressing was applied.

All operative steps were identical for all groups, with the exception of the production of the crush injury in Groups 2 and 4. In Groups 1 and 2, an inactive circuit was implanted.

\subsection{Footprint recording and analysis}

Hind foot prints were obtained on paper strips impregnated with a $1 \%$ bromophenol blue acetone solution and left to dry up, according to the method proposed by De Medinaceli and colleagues (De Medinaceli et al., 1982, 1984) and modified by Lowdon et al. (1988). The bromophenol blue impregnated paper becomes yellow after drying but turns immediately and permanently to blue when in contact with water or any water based solution. Instead of plain water the animals' hind paws were immersed into ordinary domestic detergent, which prevents excessive dispersion of the print. The animals were made to walk on the walking track where they left about three imprints of each paw. The paper strips containing the imprints were left to dry up and copied with a high-resolution scanner. The digitized imprints were then stored and analyzed in a computer with the assistance of a GRAPHIC software package specially developed for that purpose, which allowed handling of the stored imprints and automatically calculated the SFI (Oliveira et al., 2001). The parameters measured in the footprints, as indicated by previous authors (Bain et al., 1989; Carlton and Goldberg, 1986; De Medinaceli et al., 1982, 1984), were print length (PL, or the maximal distance between the tip of the longest toe and the heel), toe spread (TS, or the distance between the first and fifth toe), and intermediate toe spread (IT, or the distance between the second and fourth toe), both in the normal (N) and experimental (E) paw prints (Fig. 1). The landmarks of each parameter were simply clicked with the mouse on the monitor screen according to a previously established sequence and the SFI was automatically calculated, including a graphic plot of the regeneration curve as a function of time. The formula used to calculate the SFI

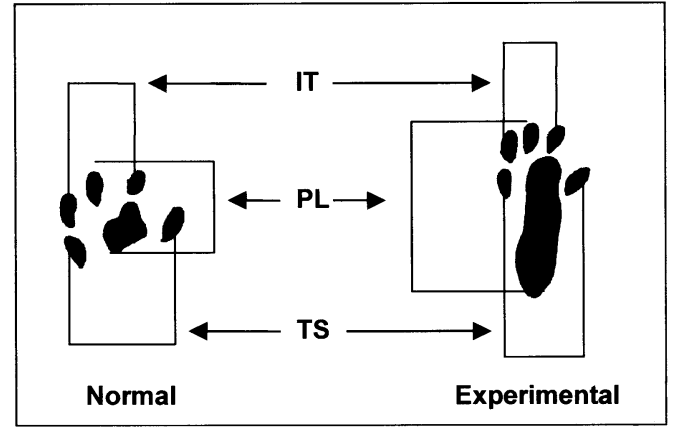

Fig. 1. Schematic drawing of the SFI parameters measured on the normal and experimental paw prints.

was that proposed by Bain et al. (1989), as follows:

$$
\begin{aligned}
\mathrm{SFI}= & -38.3 \times \frac{\mathrm{EPL}-\mathrm{NPL}}{\mathrm{NPL}}+109.5 \times \frac{\mathrm{ETS}-\mathrm{NTS}}{\mathrm{NTS}} \\
& +13.3 \times \frac{\mathrm{EIT}-\mathrm{NIT}}{\mathrm{NIT}}-8.8
\end{aligned}
$$

Hind paw prints were obtained and analyzed at weekly intervals from the first to the third week and comparisons were made between the postoperative prints from the normal and operated on paw and between preoperative and postoperative data.

\subsection{Histologic and morphometric studies}

The sciatic nerve was entirely removed through a posterolateral approach to the thigh and fixed in a $0.1 \mathrm{M}$ phosphate buffered $2.5 \%$ glutaraldehyde water solution for $2 \mathrm{~h}$ at average $6{ }^{\circ} \mathrm{C}$ in a refrigerator, after which time a $5 \mathrm{~mm}$-long segment of the distal portion of the nerve was resected, beginning $3 \mathrm{~mm}$ distal to the lesion site, and postfixed with $2 \%$ osmium tetroxyde for $12 \mathrm{~h}$. It was then dehydrated in ethilic alcohol water solution of growing concentration, from 25 to $100 \%$ at $25 \%$ increases for $1 \mathrm{~h}$ each, and embedded in epoxy resin (EPON-812) at $60{ }^{\circ} \mathrm{C}$ for $72 \mathrm{~h}, 5 \mu \mathrm{m}$-thick sections were cut and stained with $1 \%$ paraphenylenodiamine. The sections were examined with a light microscope (Zeiss Axiophoto) equipped with a video camera linked to a microcomputer loaded with the KS 400 MEASURE INTERACTIVE (version 2.0) software.

The first step of morphometric analysis consisted of capturing each individual fascicule image, followed by measuring each fascicule area by surrounding its internal epineural edge with the mouse (magnification: objective $2.5 \times$, optovar $1.6 \times$, camera $0.5 \times$ ). The next step consisted of capturing sequential inner areas of each fascicule (magnification: objective $100 \times$, optovar $1.6 \times$, camera $0.5 \times)$, followed by measuring the fiber density (quotient between number of fibers and area of counting) of each area and calculating the fiber nerve density of each fascicule (sum of all individual area 
densities). Other parameters selected for analysis were blood vessel density, average fascicule area (quotient between total fascicule area and number of fascicules), average fiber density (quotient between the sum of individual fascicule densities and number of fascicules) and $\mathrm{G}$ quotient (quotient between axone diameter and fiber diameter) for every fiber diameter interval.

Statistical analysis was carried out by Kruskal-Wallis non-parametric multiple comparison test for both SFI and morphometric studies data at the $5 \%$ significance level $(P \leq 0.05)$. Spearman correlation coeficient $(\mathrm{r})$ was used to detect any possible correlation between the values of SFI and periods of observation in Groups 2 and 4 .

\section{Results}

The clinical appearance of the hind paw was virtually normal in Groups 1 and 3 throughout the experiment, including the first postoperative days, a period during which the operative wound did not seem to interfere with gait. In Groups 2 and 4, the foot and toes remained deeply flexed and the animal was almost unable to stand on its paw up to the first postoperative week, when partial weight bearing was resumed on the operated on paw. Both appearance and weight bearing slowly improved over the next 2 weeks in Groups 2 and 4, but did not return to normal until the 21st day, when the animals were killed and the sciatic nerve removed for histologic and morphometric studies.

\subsection{Sciatic functional index}

172 hind paw prints were analyzed, including preoperative, 7 th, 14th and 21 st day records. In the control groups ( 1 and 3$)$ the imprints were very much similar to the normal ones obtained on the preoperative period and the SFI maintained stable around -10 , considered normal, all long the experiment. In Groups $2(n=9)$ and $4(n=14)$ the imprints were longer and narrower on the 7 th day than preoperatively, because the animals used both the heel and shrunken toes to stand. The average SFI in Groups 2 and 4 on the 7th day was around -100 , meaning complete functional loss, but improved to, respectively, -88.9 and -72.6 on the 14 th day, and to -50.5 and -16 on the 21 st day, the differences between these two groups being significant $(P \leq 0.05)$, with a very high correlation coefficient $(P<0.001)$ between SFI and postoperative period (Fig. 2).

\subsection{Histologic studies}

Histologic appearance was essentially normal in Groups 1 and 3, with the regular distribution of small and large diameter nerve fibers and proportion between

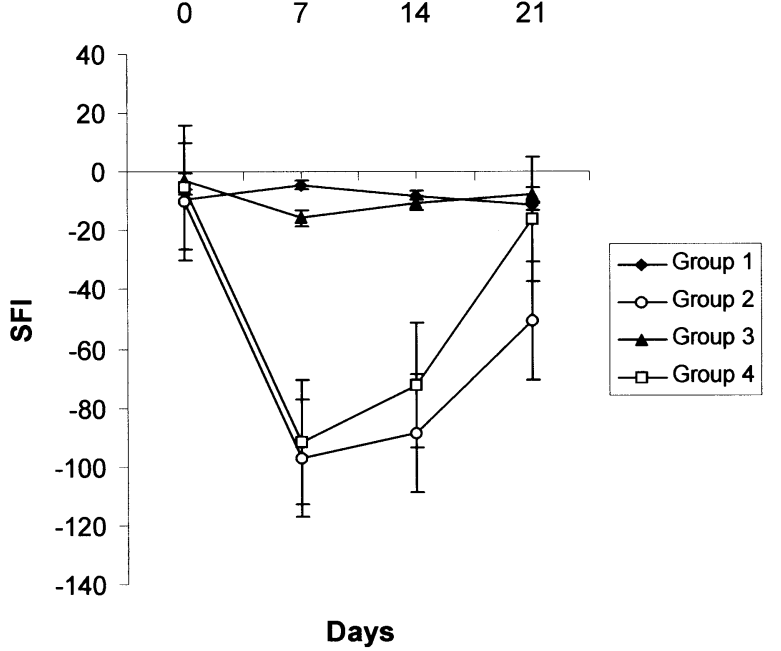

Fig. 2. Graphic of the SFI behavior according to grouping. In Groups 1 and 3, the SFI remained nearly unchanged throughout the experiment. In Group 2, the SFI evolved from a deep to a moderate dysfunction while in Group 4 it resumed a nearly normal value, from the 7 th to the 21 st postoperative day.

myelin sheath thickness and fiber diameter; intraneural blood vessels were more numerous and of larger diameter in Group 3 than in Group 1. In Group 2, Wallerian degeneration and unmyelinated fibers were still evident, but small diameter thin myelin sheath fibers predominated, with a large number of reactive Schwann cell nuclei, thus characterizing the fiber reparative process and regeneration of the myelin sheath; although present, intraneural blood vessels were less frequently seen, being also of smaller diameter than in Group 3 and 4.

In Group 4, the histologic appearance was very similar to Group 2, with many unmyelinated fibers, but small diameter thin myelin sheath fibers predominating. Wallerian degeneration was still present as well, but the reactive Schwann cell nuclei were more evident than in Group 2. However, the most stricking feature in this group was the great number of intraneural blood vessels, which were also of greater diameter than in the other groups (Figs. 3 and 4).

\subsection{Morphometric studies}

Data concerning the results of the morphometric studies are summarized in Table 1. In Group 1 (intact nerve, inactive circuit) the average myelinated fiber density was 16148 fiber $/ \mathrm{mm}^{2}$ (range: 13117-22 192 fiber $/ \mathrm{mm}^{2}$ ) with fiber diameter varying from 1.4 to $12.43 \mu \mathrm{m}$. Myelinated fiber density according to fiber diameter presented a normal distribution but fibers with diameter between 3 and $3.5 \mu \mathrm{m}$ were more numerous. The $\mathrm{G}$ quotient also presented a normal distribution with a peak at 0.6 . 

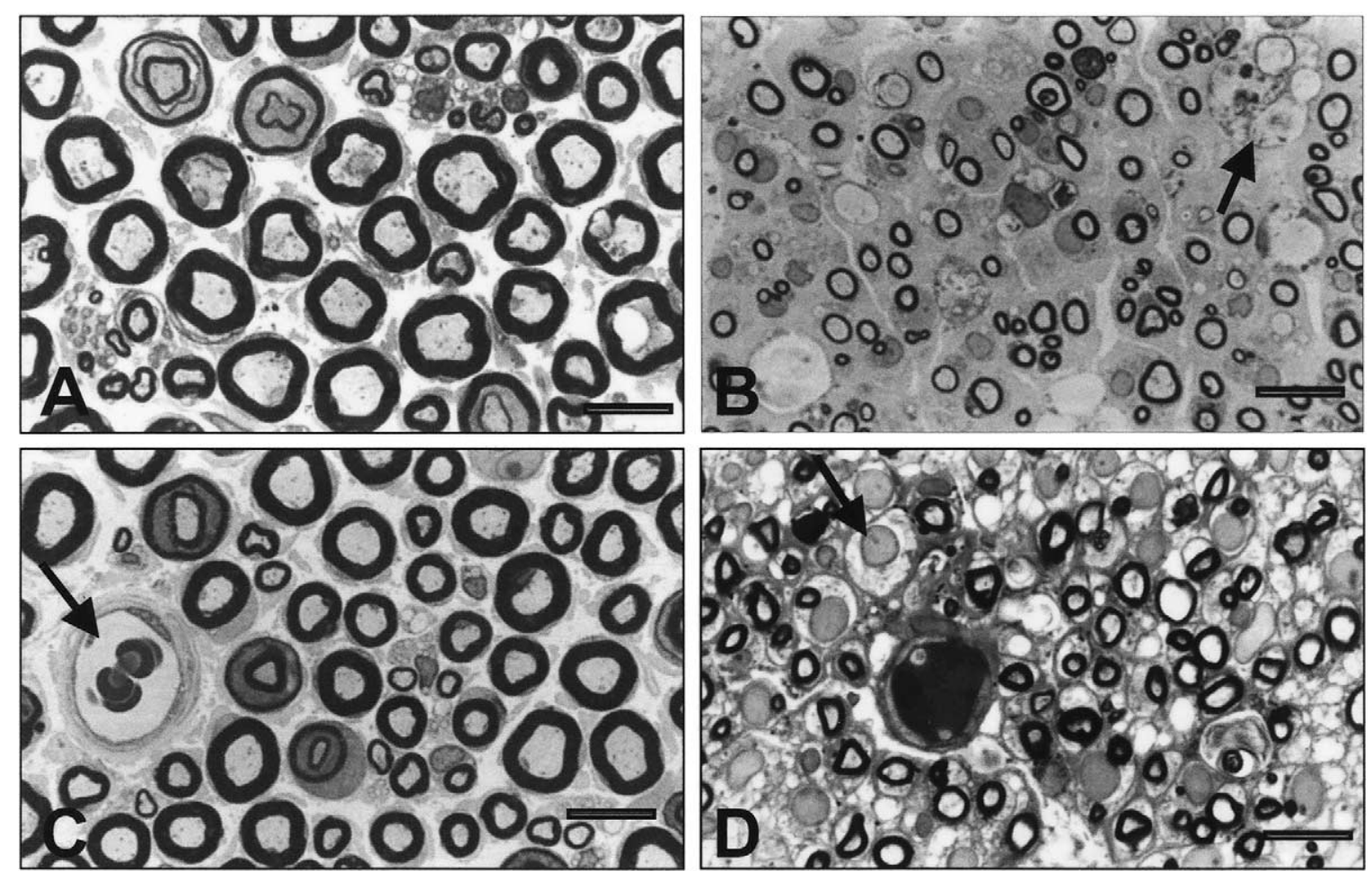

Scale bar: $10 \mu \mathrm{m}$

Fig. 3. Normal appearance of the sciatic nerve, with small and large diameter myelinated fibers, in Group 1 (A); many small diameter myelinated and unmyelinated fibers, and fibers undergoing Wallerian degeneration (arrow), in Group 2 (B); a similar appearance to A, but with a blood vessel between small and large diameter myelinated fibers (arrow), in Group 3 (C); many small diameter myelinated and unmyelinated fibers and large round Schwann nuclei (arrow), indicating a reactional process, in Group 4 (D). (Paraphenylenodiamine; scale bar $10 \mu \mathrm{m}$ ).

In Group 2 (nerve injury, inactive circuit) the average myelinated fiber density was 15669 fiber $/ \mathrm{mm}^{2}$ (range: $11647-18253 \mathrm{fiber} / \mathrm{mm}^{2}$ ) and the fiber diameter varied between 1.4 and $7.54 \mu \mathrm{m}$, with predominance of fibers between 3.0 and $3.5 \mu \mathrm{m}$ and evident right deviation on the distribution of fiber density according to fiber diameter, thus characterizing a unimodal trend of regeneration. The $\mathrm{G}$ quotient showed a right deviation with a peak at 0.7 .

In Group 3 (intact nerve, active circuit) the average myelinated fiber density was 16782 fiber $/ \mathrm{mm}^{2}$ (range: $13202-19101 \mathrm{fiber} / \mathrm{mm}^{2}$ ) and the fiber diameter varied between 1.47 and $11.66 \mu \mathrm{m}$. The distribution of fiber density according to fiber diameter tended to be bimodal, with greater density of myelinated fibers of 6.5 and $7.5 \mu \mathrm{m}$. The $\mathrm{G}$ quotient presented a normal distribution, with a peak at 0.6 .

Finally in Group 4 (nerve injury, active circuit) the average myelinated fiber density was 20294 fiber $/ \mathrm{mm}^{2}$ (range: 14923-24525 fiber $/ \mathrm{mm}^{2}$ ) with fiber diameter varying between 1.45 and $6.81 \mu \mathrm{m}$. The distribution of fiber density according to fiber diameter showed also a trend to a right deviation with a peak between 3.0 and $3.5 \mu \mathrm{m}$, although with greater density than in the previous groups, thus suggesting an increased re-myelinization. The $\mathrm{G}$ quotient presented a slightly more pronounced right deviation than in Group 2, with a small peak at 0.35 and a high peak at 0.7 (Figs. 5 and 6).

\subsection{Statistical analysis}

Myelinated fiber density was significantly different between the four experimental groups, considering all fiber diameter intervals, according to Kruskal-Wallis non-parametric test. In Group $4(n=14)$, there was a significantly higher density of small diameter fibers, particularly between 3 and $3.5 \mu \mathrm{m}(P<0.001)$ and 4 and $4.5 \mu \mathrm{m}(P=0.001)$, in comparison with all three groups. Large diameter fibers were significantly more numerous in Groups $1(n=9)$ and $3(n=10)$ than in Groups $2(n=$ $10)$ and $4(n=14)$. In Group 3 , there was a significantly higher density of fibers of $6-10.5 \mu \mathrm{m}$ in diameter $(P<$ 0.001 ), as compared with all other groups.

The analysis of myelinated fiber density according to the $\mathrm{G}$ quotient distribution did not differ significantly between groups up to the $0.45-0.5$ intervals. In the $0.5-$ 0.6 interval, density was significantly higher in Groups 1 and $3(P<0.001)$; in the $0.65-0.75$ interval, density became significantly higher in Groups 2 and $4(P<$ 0.001 ). The high density observed for Groups 2 and 4 in the $0.65-0.75$ interval probably indicates regeneration of the fiber myelin content. For Group 4 alone, the 

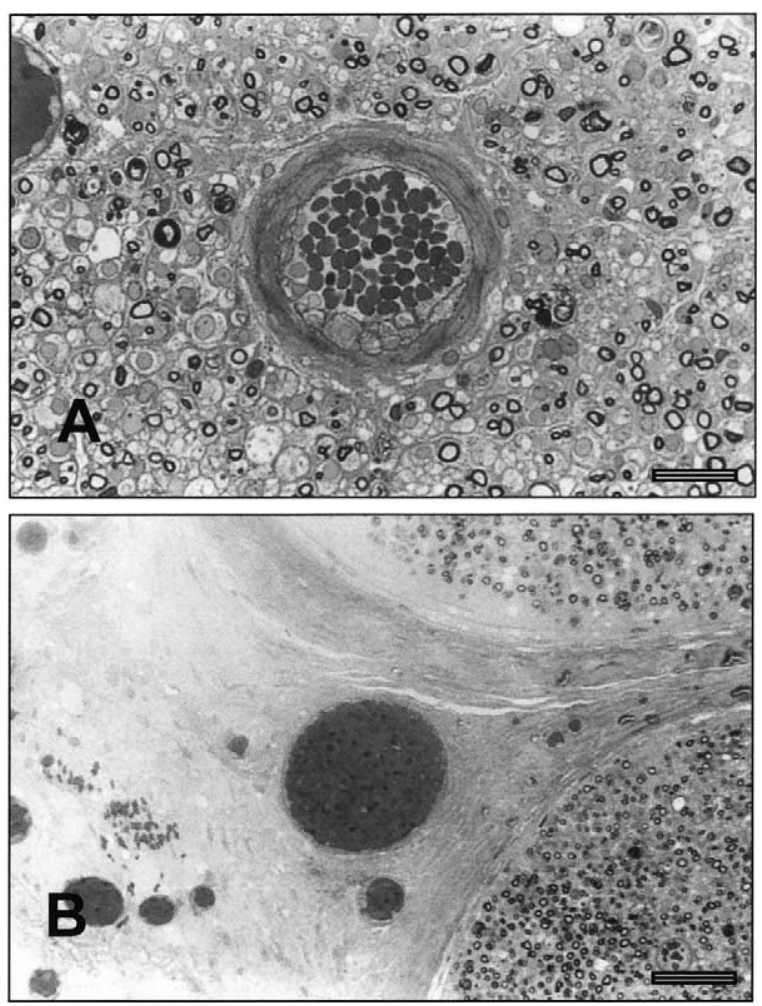

Scale bar: A) $20 \mu \mathrm{m}$; B) $50 \mu \mathrm{m}$

Fig. 4. A large intraneural blood vessel (A) and a group of small and large diameter interfascicle vessels (B), in a specimen of Group 4. (Paraphenylenodiamine; scale bar: (A) $20 \mu \mathrm{m}$, (B) $50 \mu \mathrm{m}$ ).

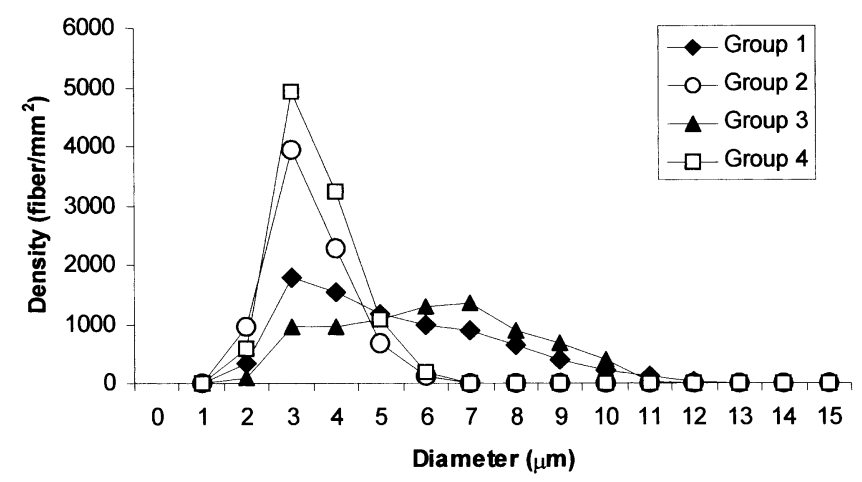

Fig. 5. Graphic of the nerve fiber density distribution according to fiber diameter in all groups, showing a trend to bimodal distribution for Groups 1 and 3 and clear unimodal distribution for Groups 2 and 4 , with great concentration of small diameter fibers.

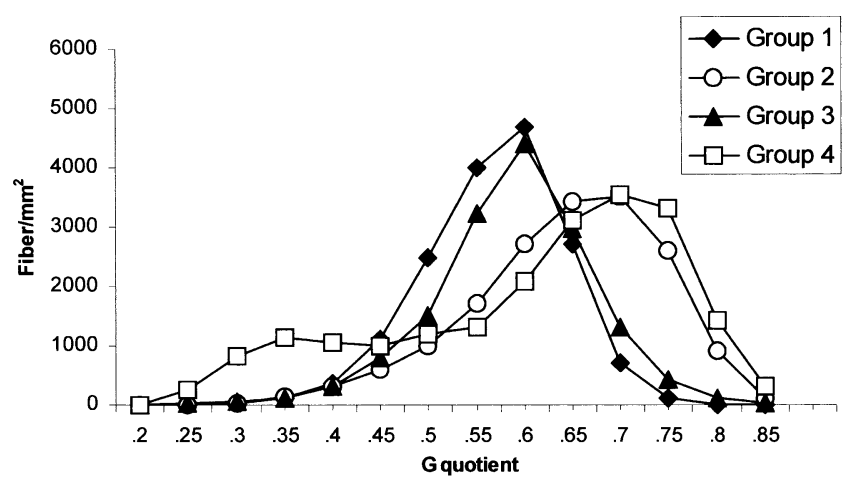

Fig. 6. Graphic of the G quotient distribution according to nerve fiber density in all groups, showing a normal distribution with a peak at 0.6 for Groups 1 and 3, and a right deviation with a peak at 0.7 for Groups 2 and 4 , but with a small peak at 0.35 for Group 4 only.

small peak observed for the $0.35 \mathrm{G}$ quotient probably indicated a delay in axonal atrophy (Fig. 6).

Blood vessels were more numerous in the groups with electric stimulation (268.4 and 245.83 vessels $/ \mathrm{mm}^{2}$ in Groups 3 and 4, respectively) than in those without electric stimulation (157.14 and 159.0 vessels $/ \mathrm{mm}^{2}$ in Groups 1 and 2, respectively), but the differences were not significant $(P=0.16)$.

\section{Discussion}

The fact that electric stimulation enhances peripheral nerve regeneration is still controversial. The purpose of the present investigation has been to correlate data from morphologic parameters of regenerating nerves with those of functional evaluation, in a model of crush injury of the sciatic nerve of rats followed by the application of a constant direct electric current. The experiment was devised to evaluate results on the short range, when differences between stimulated and nonstimulated nerves could be more evident.

Functional evaluation by the SFI method used in this experiment has already been proved to be quite reliable considering its strong correlation with morphologic evaluation. It is an indirect measurement of the rat's hind paw motor function, but it probably gives also a measure of the actual overall sciatic nerve function, since the paw's motor function during gait depends on

Table 1

General morphometric data of groups 1 through 4

\begin{tabular}{lllll}
\hline & Group 1 & Group 2 & Group 3 & Group 4 \\
\hline Fiber density (average \pm S.D.) & $16148 \pm 3948$ & $15669 \pm 2237$ & $16781 \pm 2204$ & $20294 \pm 3770$ \\
Axon diameter range & $1.40-12.43$ & $1.40-7.54$ & $1.47-11.66$ & $1.45-6.81$ \\
Axon diameter peak & $3.0-3.5$ & $3.0-3.5$ & $6.5-7.5$ & $3.0-3.5$ \\
G quotient peak & $0.55-0.60$ & $0.65-0.75$ & $0.55-0.60$ & $0.70-0.75$ \\
\hline
\end{tabular}


plantar sensitivity as well. As a negative indicator of the degree of nerve dysfunction, the SFI may vary from zero (normal function or absence of dysfunction) to -100 (complete dysfunction). The SFI is calculated according to a previously tested relatively complex mathematics formula, which correlates well with the clinical appearance of the paws and includes all possible permutations (Bain et al., 1989; Oliveira et al., 2001). Neverthless, in the present investigation the SFI was never zero for the rats with an intact sciatic nerve as it was expected to be, but oscillated around -10 , probably indicating that the method is not entirely precise. However, our SFI figures along time clearly showed that there was a trend to functional recovery in the groups with a crush injury, whether stimulated or not, and that such a recovery was better in the group with electrical stimulation. Actually, the SFI for Group 4 (crush injury, electric stimulation) was close to that for the control groups, with an intact nerve ( 1 and 3 ) on the 21st postoperative day, while in Group 2 (crush injury, no electric stimulation) it was still too low (around -50), compatible with a moderate dysfunction of the sciatic nerve (Fig. 2).

The results of the morphometric evaluation confirmed that morphologic regeneration is an ongoing process on the 21st postoperative day, as described earlier by Mira (1979), who observed an increased population of myelinated nerve fibers as early as the second week. In the present study, nerve fiber density was about 5\% lower than normal (about $16465 \mathrm{fiber} / \mathrm{mm}^{2}$ ) in Group 2, but about $23 \%$ higher in Group 4. In a previous publication, we demonstrated a spontaneous increase in nerve fiber density following a crush injury of the sciatic nerve, which was indirectly proportional to the degree of nerve injury caused by growing loads $(100,500$ and $15000 \mathrm{~g}$ ), so that the most severe injury enhanced a decreased nerve fiber density compared with a slight $(100 \mathrm{~g})$ or moderate $(500 \mathrm{~g})$ injury and to normal. The fact that the SFI improves together with the recovery of nerve fiber density with time is not merely coincidental but means that function is directly linked to the degree of morphologic regeneration (Oliveira et al., 2001). In the present investigation, the same heavy load was used to produce a severe crush injury to the sciatic nerve, but nerve fiber density increased with electric stimulation. Considering that the only differential factor between Groups 2 and 4 was electric stimulation, it seems clear to us that this stimulation positively influenced nerve regeneration. Furthermore, a slightly (4\%) increased nerve fiber density was also observed following the application of electric stimulation onto an intact nerve (Group 3), thus suggesting that electric stimulation can alter morphologic parameters of intact nerves, with possible but unknown effects on function.

On the other hand, in both Groups 2 and 4, there was a clear predominance of small diameter fibers (around 3 $\mu \mathrm{m}$ in diameter, compared with the wider distribution from 3 to $7 \mu \mathrm{m}$ observed in the intact nerves of Groups 1 and 3 ) in a trend to unimodal regeneration (Fig. 5). So, both the increased nerve fiber density and the decreased average nerve fiber diameter observed here can have only resulted from nerve sprouting and this may well be one of the mechanisms by which electric stimulation enhances nerve regeneration.

Low G quotient values (around 0.4) usually indicate axonal degeneration, while high values (around 0.7) indicate either myelin degeneration or regeneration. In this investigation, two peaks were observed for Group 4, the first at 0.35 and the second at 0.7 (Fig. 6) and such events were interpreted as delayed axonal degeneration and stimulated myelin regeneration, respectively. Myelin regeneration may be considered a spontaneous process, since it was observed also in Group 2, but delayed axonal degeneration was only observed in Group 4 and may well be an effect of electric stimulation.

Any peripheral nerve lesion is followed by axon membrane collapse, axoplasm outflow and intense ion exchange, extracellular ions $\left(\mathrm{Na}^{+}, \mathrm{Ca}^{+}\right)$entering the fiber and intracellular ions $\left(\mathrm{K}^{+}\right)$leaking out, in consequence of which the axoplasm would become more positive. The intracell components like the mitochondrion migrate toward the lesion site, those in the proximal segment moving distally and those in the distal segment, proximally, that is from a less positive toward a more positive environ. The application of an exogenous electric current with the cathode placed distally from the lesion site would enhance the regeneration process, first by stopping the calcium ions from getting into the axon and second, by generating a counter-current into the terminal axon, whose lesser electric tissue resistance makes it more permeable to the electric current flow. Therefore, the exogenous current directed from proximal to distal would oppose the endogenous current, which flows in the distal-proximal direction inside the axon and this would preserve the axoplasm transport and contribute to regeneration (Borgens et al., 1989). The larger number of atrophic fibers (axonal degeneration) observed in Group 4 compared with Group 2 seems to confirm that somehow the axonal flow was maintained despite the severity of the damage inflicted by crushing, probably confirmed by the $0.35 \mathrm{G}$ quotient peak discussed above.

Furthermore, the morphologic changes observed in Group 4 showed that electric stimulation seems to interfere also with the supporting structures of the peripheral nerves, particularly blood vessels and Schwann cells, whose number was increased in this group.

It was our conclusion that low intensity directly applied electric stimulation enhances both morphologic and functional regeneration of the crushed sciatic nerve of rats in experimental conditions, probably by delaying axonal degeneration, stimulating nerve sprouting and 
accelerating myelin sheath regeneration. Electric stimulation of nerve regeneration has a potential for application in humans.

\section{Acknowledgements}

The authors acknowledge Professor Amilton Antunes Barreira, from the Department of Neurology and Psychiatry for his kind permission to use the Laboratory of Neurosciences facilities and equipments, and $\mathrm{Mr}$ Antonio Renato Meirelles e Silva and Ms Maria Cristina Lopes Schiavoni for their kind technical assistance.

\section{References}

Bain JR, Mackinnon SE, Hunter DA. Functional evaluation of complete sciatic, peroneal, and posterior tibial nerve lesions in the rat. Plast Reconstr Surg 1989;83(1):129-36.

Behse F. Morphometric studies on the human sural nerve. Acta Neurol Scand, Suppl 1990;132(82):5-38.

Beveridge JA, Politis MJ. Use of exogenous electrical current in the treatment of delayed lesions in peripheral nerves. Plast Reconstr Surg 1988;82(4):573-7.

Borgens RB, Robinson KR, Vanable JW, McGinnis ME. Electric field in vertebrate repair. New York: Alan R. Liss, Inc, 1989:77-170 (Chap 3 and 4).

Brighton CT. Current concepts review the treatment of non-unions with electricity. J Bone Joint Surg 1981;63A:847-51.

Carlton JM, Goldberg NH. Quantitating integrated muscle function following reinervation. Surg Forum 1986;37:611-2.

De Medinaceli L, Freed WJ, Wyatt RJ. An index of the functional condition of rat sciatic nerve based on measurement made from walking tracks. Exp Neurol 1982;77:634-43.

De Medinaceli L, Derenzo E, Wyatt RJ. Rat sciatic functional index data management system with digitized input. Comput Biomed Res 1984;17:185-92.
Gutmann, E., Gutmann, L., 1942. Factors affecting recovery of sensory function after nerve lesions. J. Neurol. Psychiat. 5, 117129

Kerns JM, Fakhouri AJ, Weinrib HP, Freeman JA. Effects of D.C. electrical stimulation on nerve regeneration in the rat sciatic nerve. Anat Rec 1986;214:64A.

Kerns JM, Pavkovic IM, Fakhouri AJ, Wickersham KL, Freeman JÁ. An experimental implant for applying a DC electrical field to peripheral nerve. J Neurosci Methods 1987;19:217-23.

Kerns JM, Fakhouri AJ, Weinrib HP, Freeman JÁ. Electrical stimulation of nerve regeneration in the rat: the early effects evaluated by a vibrating probe and electron microscopy. Neuroscience 1991;40(1):93-107.

Lowdon IMR, Seaber AV, Urbaniak JR. An improved method of recording rat tracks for measurement of the sciatic functional index of De Medinaceli. J Neurosci Methods 1988;24:279-81.

McDevitt L, Fortner P, Pomeranz B. Application of weak electric field to the hindpaw enhances sciatic motor nerve regeneration in the adult rat. Brain Res 1987;416:308-14.

Mira JC. Experimental study using a direct current electrical field to promote peripheral nerve regeneration. J Reconstr Microsurg 1995;11(3):189-93.

Oliveira EF, Mazzer N, Barbieri CH, Selli M. Correlation between functional index and morphometry to evaluate recovery of the rat sciatic nerve following crush injury: experimental study. J Reconstr Microsurg 2001;17(1):69-75.

Politis MJ, Zanakis MF, Albala BJ. Mammalian optic nerve regeneration following the application of electric fields. J Trauma 1988a;28(11): $1548-52$.

Politis MJ, Zanakis MF, Albala BJ. Facilitated regeneration in the rat peripheral nervous system using applied electric field. J Trauma 1988b;28(9):1375-81.

Pomeranz B, Campbell J. Weak electric current accelerates motoneuron regeneration in the sciatic nerve of 10 -month-old rats. Brain Res 1993;603:271-8.

Pomeranz B, Mullen M, Markus H. Effect of applied electrical fields on sprouting of intact saphenous nerve in adult rat. Brain Res 1984;303:331-6.

Roman GC, Strahlendorf HK, Coates PW, Rowley BA. Stimulation of sciatic nerve regeneration in the adult rat by low-intensity electric current. Exp Neurol 1987;98:222-32.

Shen N, Zhu J. Experimental study using a direct current electrical field to promote peripheral nerve regeneration. J Reconstr Microsurg 1995;11(3):189-93. 